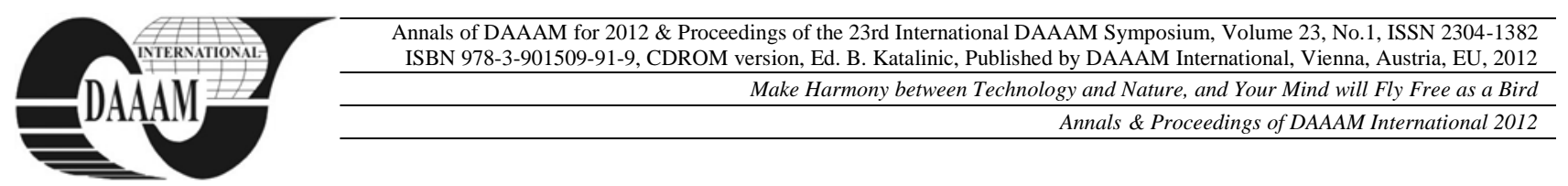

\title{
APPLICATIONS OF RAPID PROTOTYPING FUSED DEPOSITION MODELING MATERIALS
}

\author{
NOVAKOVA - MARCINCINOVA, L[udmila] \& NOVAK - MARCINCIN, J[ozef]
}

\begin{abstract}
Fused Deposition Modeling (FDM) represents one from basic Rapid Prototyping (RP) methods used in industrial practice. In this contribution are presented basic information about common and advanced materials used for realization of products by Fused Deposition Modeling rapid prototyping technology application. In basic Rapid Prototyping devices the initial state of material can come in either solid, liquid or powder state. In solid state material can be in form pellets, wire or laminates. The current range materials include paper, nylon, wax, resins, metals and ceramics. Directly in FDM technology are used as basic material Acrylonitrile Butadiene Styrene, polyamide, polycarbonate, polyethylene and polypropylene. In special FDM applications can be used silicon nitrate, aluminium oxide, hydroxypatite and stainless steel for a variety of structural, electroceramic and bioceramic applications. In final part of the article is described design and production of experimental gearbox realized on workplace of the authors.

Keywords: rapid prototyping, fused deposition modeling, FDM materials, ABS plastic, experimental gearbox
\end{abstract}

\section{INTRODUCTION}

Rapid Prototyping (RP) of physical parts or otherwise known as solid freeform manufacturing or desktop manufacturing or layer manufacturing technology, represents the new phase in the evolution of prototyping. The invention of this series of Rapid Prototyping methodologies is described as a watershed event because of the tremendous time savings, especially for complicated model. Through the parts (individual components) are relatively three times as complex as parts made in 1970s, the time required to make such a part now averages only three weeks. Since 1988, more then twenty different rapid prototyping techniques have emerged. Rapid Prototyping is the automatic construction of physical objects using additive manufacturing technology. The first techniques for Rapid Prototyping became available in the late 1980 s and were used to produce models and prototype parts. Today, they are used for a much wider range of applications and are even used to manufacture production-quality parts in relatively small numbers. The use of additive manufacturing for Rapid Prototyping takes virtual designs from computer aided design (CAD), transforms them into thin, virtual, horizontal cross-sections and then creates successive layers until the model is complete. The primary advantage to additive fabrication is its ability to create almost any shape or geometric feature [2].

\section{BASIC RAPID PROTOTYPING METHODS}

Rapid Prototyping has also been referred to as solid free-form manufacturing, computer automated manufacturing and layered manufacturing. RP has obvious use as a vehicle for visualization. In addition, RP models can be used for testing, such as when an airfoil shape is put into a wind tunnel. RP models can be used to create male models for tooling, such as silicone rubber moulds and investment casts. In some cases, the RP part can be the final part, but typically the RP material is not strong or accurate enough. When the RP material is suitable, highly convoluted shapes (including parts nested within parts) can be produced because of the nature of RP. There are a multitude of experimental RP methodologies either in development or used by small groups of individuals. This section will focus on RP techniques that are currently commercially available, including Stereolithography (SLA), Selective Laser Sintering (SLS), Laminated Object Manufacturing (LOM), Fused Deposition Modeling (FDM), Solid Ground Curing (SGC) and Ink Jet printing techniques.

Stereolithography is the name of a process used to make three-dimensional models from computer databases. Designers in manufacturing companies use the technique extensively to assist them in visualizing a plan for a new car part, an airplane component, or a small medical device. By using a series of photochemical reactions and a unique assembling system, designers can quickly replicate a computer representation as a plastic model or prototype. The prototype provides a simple visualization of what a part will actually look like in three dimensions. Stereolithography uses a photochemical event, or more specifically, it uses small lasers and a series of photochemical events, to direct the formation of points of plastic on the surface of an easily solidified liquid. The computer directs a laser across the surface of a polymerizable plastic (to polymerize means to make large molecules from small ones - all common plastics are large, non-homogeneous molecules or polymers) converting the polymerizable material point by point into a solid. This is done, in this particular experimental setup, on a platform that is held a few millimeters under the surface of the photoreactive liquid. After the laser has converted to solid plastic all the points in one layer that are needed for the model, producing a two dimensional lithograph of fixed $\mathrm{z}$ dimension, the platform is dropped a small increment and a second layer is built on top of the first. This process continues until each layer of the computer graphic, or plan, is converted to solid plastic. When the process is complete the model can be lifted from the plastic vat as an exact replica of the computer graphic [3].

Selective Laser Sintering can be used in every stage of the product development cycle, from the production of one-shot models to functional test parts and small 
production series. Laser Sintering is also a technique by which parts are built layer by layer. The basic material consists of powder with particle sizes in the order of magnitude of $50 \mu \mathrm{m}$. Successive powder layers are spread on top of each other. After deposition, a computer controlled $\mathrm{CO}_{2}$ laser beam scans the surface and selectively binds together the powder particles of the corresponding cross section of the product. During laser exposure, the powder temperature rises above the glass transition point after which adjacent particles flow together. This process is called sintering. Being a solid material, the powder has the attractive feature of being self-supporting for the generated product sections. This makes supports (typical for Stereolithography) redundant. The polyamide (PA) material allows the production of fully functional prototypes with high mechanical and thermal resistance. Polyamide parts have an excellent long-term stability and are resistant against most chemicals. They can be made watertight by impregnation. The PA material is certified as biocompatible, foodsafe and not harmful to health or the environment. The use of PA powder filled with glass particles (PA-GF) has a much higher thermal resistance and is typically used in functional tests with high thermal loads. Alumide is a blend of aluminium powders and PA powder, which allows metallic-looking, non-porous components to be machined easily and is resistant to high temperatures. A typical application for Alumide is the manufacture of stiff parts of metallic appearance for applications in automotive manufacture, for small production runs and jig manufacture, among other aspects $[3,8]$.

Laminated Object Manufacturing (LOM) procedure is used to manufacture a prototype by lamination and laser finishing (cutting) of materials such as paper, polymeric films and foils, and metal laminates. With polymeric foils better mechanical properties are achieved than with paper. The sheets are laminated into solid blocks by adhesion joining, clamping and ultrasonic welding. Using heat and pressure each sheet, foil or paper is adhered to the block and a new layer is formed. The material is supplied by means of a roller on one side of the machine and taken to another side. The heated roller provides pressure and heat necessary for the new layer to be glued to the already produced prototype part. The working platform is lowered for the foil thickness, which is usually a thickness of $0,07 \mathrm{~mm}$ to $0,2 \mathrm{~mm}$. After the layer (foil) has been deposited, the laser beam or knife cuts a part of the material into the form of the finished product. Usually $\mathrm{CO}$ laser of $25 \mathrm{~W}$ or $50 \mathrm{~W}$ power is used. Standard laminated object manufacturing procedure use film on which a layer of glue is applied, which is then cut by a knife into adequate form. Then, an "antiglue" layer is applied on certain places where there is no prototype, i.e. the glue is neutralized. The next film layer is applied and it is glued to the previous one and the prototype is manufactured all the way to the final layer. As in other procedures, the process starts with the lower transverse section. The presence of the auxiliary material around the prototype has its advantages and drawbacks. First, the external support structure is not necessary. In prototype manufacturing inside a supporting material, the entire geometry during manufacturing is protected against deformation due to its proper mass. LOM avoids the need to design special supporting structures that hold the isolated contours. Removal of unnecessary material after the prototype has been manufactured is far from an easy task. Careful manual cleaning procedure is necessary not to damage the sensitive parts and to insure that only the excessive material is removed. Also, the majority of materials used in case of LOM procedure do not contribute to the prototype itself. It remains with continuous strip (foil) or finishes as supporting material which is discarded after construction [9].

\section{FUSED DEPOSITION MODELING}

The Fused Deposition Modeling (FDM) process from Stratasys produces prototype parts out of Acrylonitrile Butadiene Styrene (ABS) plastic. FDM deposits a molten filament of $\mathrm{ABS}$ in a criss-cross manner resulting in direction dependant, or anisotropic, material properties. The FDM process works as follows. First, a three dimensional solid model must be created. This can be accomplished in many of the commonly available CAD packages. The model is then exported to the FDM Catalyst software using the stereolithography (STL) format. This format tessellates the part into a set of triangles. The advantage of the STL format is that most CAD systems support it, and it simplifies the part geometry by reducing it to its most basic components. The disadvantage is that the part loses some resolution, because only triangles, and not true arcs, splines, etc., now represent the geometry. However, the errors introduced by these approximations are acceptable as long as they are less than the inaccuracy inherent in the manufacturing process. Once the STL file has been exported to Catalyst, it is horizontally sliced into many thin sections. These sections represent the twodimensional contours that the FDM process will generate which, when stacked upon one another, will closely resemble the original three-dimensional part. This sectioning approach is common to all currently available $\mathrm{RP}$ processes. The software then uses this information to generate the process plan that controls the FDM machine's hardware (Fig. 1).

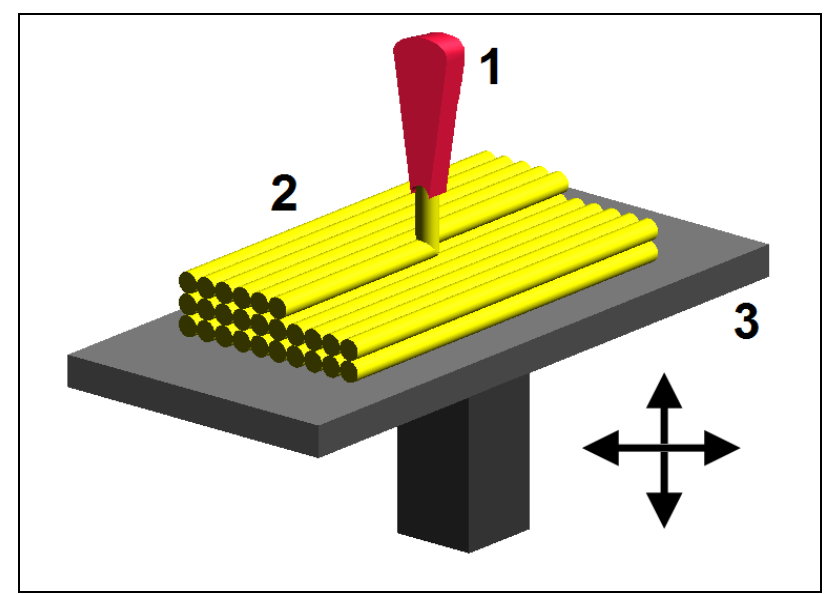

Fig. 1. Schematic model of Fused Deposition Modelling technology (1nozzle, 2-building material, 3-movable table)

In the physical process of fabrication, an ABS filament is fed through a heating element and becomes semi-molten. The filament is then fed through a nozzle and deposited onto the partially constructed part. Since the material is extruded in a semi-molten state, the newly deposited material fuses with adjacent material that has already been deposited. The head then moves around in 
the $X-Y$ plane and deposits material according to the part geometry. The platform holding the part then moves vertically in the $Z$ plane to begin depositing a new layer on top of the previous one. After a period of time, usually several hours, the head will have deposited a full physical representation of the original $\mathrm{CAD}$ file.

The FDM machine possesses a second nozzle that extrudes support material and builds support for any structure that has an overhang angle of less than $45^{\circ}$ from horizontal as a default. If the angle is less than $45^{\circ}$, more than one-half of one bead is overhanging the contour below it, and therefore is likely to fall [1].

\section{FDM MATERIALS}

Fused Deposition Modeling is one of the typical RP processes that provide functional prototypes of ABS plastic. FDM produces the highest-quality parts in Acrylonitrile Butadiene Styrene (ABS) which is a common end-use engineering material that allows you to perform functional tests on sample parts. FDM process is a filament based system which feeds the material into the heated extrusion head and extruding molten plastic that hardens layer-by-layer to form a solid part. FDM parts are tougher and more durable than those produced by SLA. ABS parts are sufficiently resistant to heat, chemicals, and moisture that allows FDM parts to be used for limited to extensive functional testing, depending upon the application [6].

FDM materials allow you to manufacture real parts that are tough enough for prototyping, functional testing, installation, and most importantly - end use. Real production thermoplastics are stable and have no appreciable warpage, shrinkage, or moisture absorption, like the resins (and powders) in competitive processes. Because thermoplastics are environmentally stable, part accuracy (or tolerance) doesn't change with ambient conditions or time. This enables FDM parts to be among the most dimensionally accurate. Basic FDM materials:

1. ABS - An ABS prototype has up to $80 \%$ of the strength of injection moulded ABS meaning that it is extremely suitable for functional applications.

2. $\mathrm{ABSi}$ - ABSi is an ABS type with high impact strength. The semi-translucent material used to build the FDM parts is USP Class VI approved.

3. ABS-M30 - ABS-M30 is $25-75 \%$ stronger than the standard ABS material and provides realistic functional test results along with smoother parts with finer feature details.

4. ABS-ESD7 - ABS-ESD7 is a durable and electrostatic dissipative material suited for End-use components, Electronic products, Industrial equipment and Jigs and fixtures for assembly of electronic components.

5. PC-ABS - PC-ABS is a blend of polycarbonate and ABS plastic which combines the strength of $\mathrm{PC}$ with the flexibility of ABS.

6. PC-ISO - PC-ISO blends are widely used throughout packaging and medical device manufactures. The PCISO material used to build the FDM parts is USP Class VI approved and also ISO 10993-1 rated.

7. ULTEM 9085 - ULTEM 9085 is a pioneering thermoplastic that is strong, lightweight and flame retardant. The ULTEM 9085 material opens up new opportunities for the direct additive construction of production grade components [11].

Very little effort seems to have been made to develop metallic materials for the FDM process. Work has been in progress in some universities and research institutions to develop new metallic and ceramic materials for rapid fabrication of functional components by FDM with higher mechanical properties. Rutgers University in the United States have carried out considerable work in the development of fused deposition of ceramics (FDC) and metals. They have used the process to fabricate functional components of a variety of ceramic and metallic materials such as silicon nitrate, PZT, aluminium oxide, hydroxypatite and stainless steel for a variety of structural, electroceramic and bioceramic applications. They create such components on the FDM using ceramic powders mixed with organic binder system. The properties of the mixed feedstock filament meet the flexibility, stiffness, and viscosity required for successful FDM processing. But the fabricated green parts need to undergo further processing to remove the organic binder and are subjected to sintering to achieve densification. Sintered part may be infiltrated with other type of metal materials. Work has also been carried out at Advanced Ceramics Research [5] for part fabrication in ceramics using the FDM process. Researchers at Virginia Tech have developed a new high performance thermoplastic composite for FDM, involving thermotropic liquid crystalline polymers (TLCP) fibres, and have used it in FDM system to fabricate prototype parts. The tensile modulus and strength of this material were approximately four times those of ABS. Therefore, prototypes fabricated with these materials would have greater functionality than those fabricated with ABS. The FDM technology thus offers the potential to produce the functional parts with a variety of materials including composite materials. But little work seems to have been done in the development of metal/polymer composites for direct rapid tooling application using the FDM system. Direct rapid tooling of injection moulding dies and inserts can be conveniently performed if a strong metal based feedstock material is available for the FDM rapid prototyping systems [7].

\section{APPLICATION OF FDM MATERIALS}

In order to minimize the prototype it was decided on the Faculty of Manufacturing Technologies of the TU Kosice with a seat in Presov, Slovakia to model the small four-graded gearbox with reverse operation and self-shift shown in Fig. 2. We did not want to design and produce any unusable parts with large surface breakdown to put it to make measurements but the prototype which would itself perform a function and was suitable for visualizing the accuracy of the printing. Because the teeth presented most accurate part of the draft, we decided to generate them using the Design Accelerator module included in Autodesk Inventor Professional 2009. This step is necessary to enter the desired parameters of gearing as a module, the distance axis, gear ratio, width of teeth and after conversion to generate a 3D model of the sprocket, which can be further modified. Model gearing was subsequently modified in $\mathrm{CAD} / \mathrm{CAM} / \mathrm{CAE}$ system CATIA V5 R19. Transfer of models between Inventor and Catia system was implemented using the exchange format IGES where they were treated [5]. 


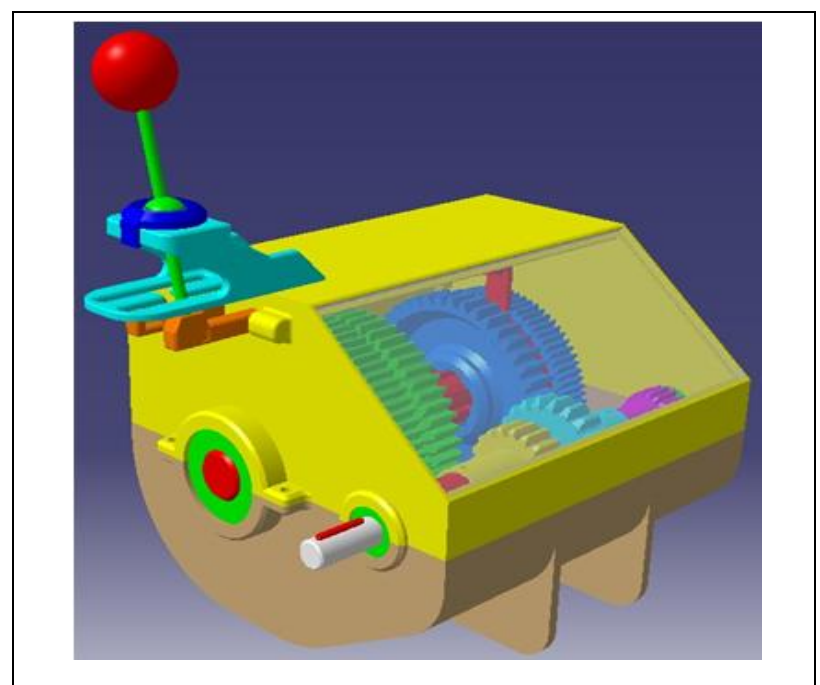

Fig. 2. CAD model of experimental gearbox

On the Department of Manufacturing Technologies there is UPrint FDM device from Dimension available. printing jet and whole work area for working temperature. This lasts about 15 minutes, during which the nozzle and purifying device are calibrated. Followed by the print itself, the nozzle is moving over $X$ - Y pad and working in the $\mathrm{Z}$ axis. After printing it is necessary to separate the support material from the building one. In the semi-simple components the support material can be separated without any problems, as because of reducing temperature it is particularly fragile. However, for complex parts with cavities there is need to use the washer to remove support material from places that are not accessible for any instrument. The last step is gear assembly, which consists of forty parts and testing of prototype functionality. During the functional testing, we used an electric motor with a speed regulator connected to the input shaft. The test showed flawless shifting and fixing of rates in the desired position [4].

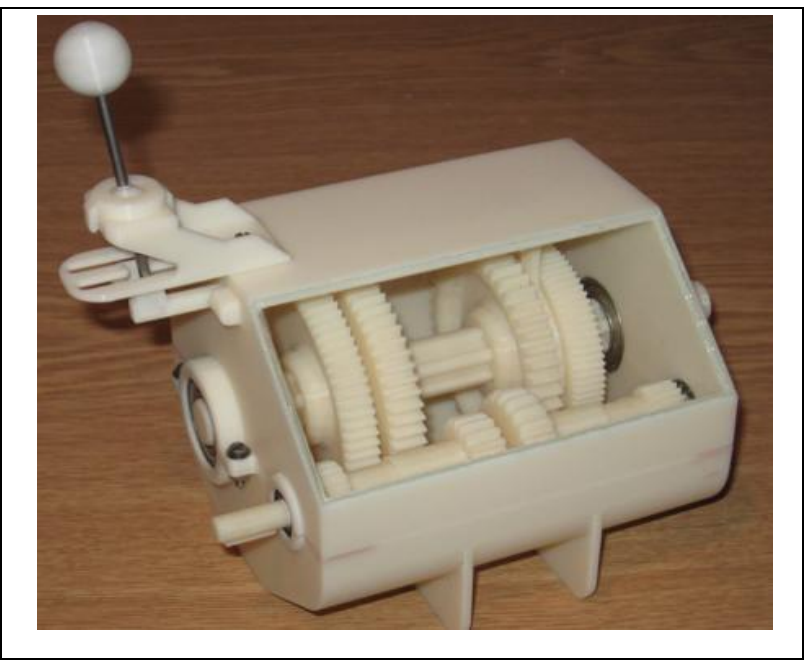

Fig. 3. Assembled prototype of experimental gearbox

\section{CONCLUSION}

In industrial practice there are currently more than 20 vendors for RP systems, the method employed by each vendor can be generally classified into the following categories: photo-curing, cutting and glueing/joining, melting and solidifying/fusing and joining/binding. Photo-curing can be further divided into categories of single laser beam, double laser beams and masked lamp. The initial state of material in Rapid Prototyping technologies can come in either solid, liquid or powder state. In solid state, it can come in various forms such as pellets, wire or laminates. The current range materials include paper, nylon, wax, resins, metals and ceramics. Most of the RP parts are finished or touched up before they are used for their intended applications. Applications can be grouped into design engineering, analysis and planning and tooling and manufacturing. A wide range of industries can benefit from RP and these include automotive, aerospace, biomedical, consumer, electrical and electronic products [10].

\section{ACKNOWLEDGEMENTS}

Ministry of Education, Science, Research and Sport of SR supported this work, contract VEGA No. 1/0032/12, KEGA No. 002TUKE-4/2012 and ITMS project 26220220125 .
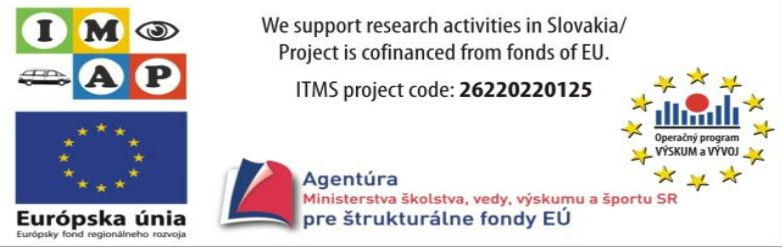

\section{REFERENCES}

[1] Ahn, S. H.; Montero, M.; Odell, D.; Roundy, S. \& Wright, P. K. (2002). Anisotropic material properties of fused deposition modeling ABS. Rapid Prototyping, Vol. 8, No. 4, pp. 248-257

[2] Barna, J.; Janak, M. \& Fecova, V. (2010). Technical and economical charakteristics of 3D printing In: Progressive technologies of cutting. FVT TU, Presov, pp. 67-74

[3] Chua, C. K.; Leong, K. F. \& Lim, C. S. (2003). Rapid Prototyping: Principles and Applications. World Scientific Publishing, Singapore, 420 p., ISBN 981-238-117-1

[4] Marcincin, J. N.; Barna, J.; Marcincinova, L. N. \& Fecova, V. (2011). Analyses and Solutions on Technical and Economical Aspects of Rapid Prototyping Technology. Tehnical Gazette, Vol. 18, No. 4, pp. 657-661, ISSN 1330-3651

[5] Marcincinova, L. N.; Barna, J.; Fecova, V.; Janak, M. \& Marcincin, J. N. (2011). Intelligent Design of Experimental Gearbox with Rapid Prototyping Technology Support. In: Proceedings of 15th Int. Conference on Intelligent Engineering Systems, Poprad, p. 77-80, ISBN 978-1-4244-8955-8

[6] Marcincinova, L. N.; Fecova, V.; Marcincin, J. N.; Janak, M. \& Barna, J. (2012). Effective Utilization of RP Technology. Materials Science Forum, Vol. 713, p. 61-66, ISSN 1662-9752

[7] Massod, S. H. \& Song, W. Q. (2004). Development of new metal/polymer materials for RT using Fused deposition modeling. Materials \& Design, Vol. 25, No. 7, p. 587-594, ISSN 0261-3069

[8] Pacurar, R.; Balc N.; Berce P. \& et al. (2008). Research on Improving the Mechanical Properties of the SLS Metal Parts. In: Annals of DAAAM for 2008 \& Proceedings of the 19th Int. DAAAM Symposium, Trnava, p. 1003-1004

[9] Pilipovic, A.; Raos, P. \& Sercer, M. (2011). Experimental testing of quality of produced by Laminated Object Manufacturing. Technical Gazette, Vol. 18, No. 2, pp. 253-260, ISSN 1330-3651

[10] Plancak, M. (2009). Rapid Prototyping \& Rapid Tooling. FTN Publishing, Novi Sad, 164 p., ISBN 978-86-7892-232-9

[11] FDM: Materials \& datasheets http://www.materialise.com/fdm-materials

(2012)

12] Lombardi IL, Hoffman RA, Waters IA, Popovich D, Souvenir C, Boggavarapu B. (1997) Issues associated with EFF \& FDM ceramic filled feedstock formulation. In: Proceedings of Solid Freeform Symposium, University of Texas, Austin, August 1997. p. 11-3 